\title{
ANTIEIGENVALUE INEQUALITIES IN OPERATOR THEORY
}

\author{
MORTEZA SEDDIGHIN
}

Received 15 March 2004

\begin{abstract}
We will prove some inequalities among trigonometric quantities of two and three operators. In particular, we will establish an inequality among joint trigonometric quantities of two operators and trigonometric quantities of each operator. As a corollary, we will find an upper bound and a lower bound for the total joint antieigenvalue of two positive operators in terms of the smallest and largest eigenvalues of these operators.
\end{abstract}

2000 Mathematics Subject Classification: 47C99.

1. Introduction. Recall that an operator $T$ is accretive if the numerical range of $T$ is a subset of the right half-plane. For an accretive operator on a Hilbert space $H$, the first antieigenvalue of $T, \mu_{1}(T)$, is defined by Gustafson to be

$$
\mu_{1}(T)=\inf _{\substack{T f \neq 0 \\\|f\|=1}} \frac{\operatorname{Re}(T f, f)}{\|T f\|}
$$

(see $[1,3,4])$. The quantity $\mu_{1}(T)$ is also denoted by $\cos T$ or $\cos _{\mathbb{R}} T$ and is called the cosine (or real cosine) of $T$. The first antieigenvalue, $\mu_{1}(T)$, has important applications in the study of the numerical range of operators. For instance, it is proved in [1] that for two positive selfadjoint operators $A$ and $B$, the product $A B$ is accretive if $\sin A \leq \cos B$. It is shown in [2] that if for a positive operator $T$ we define

$$
\sin T=\inf _{\in>0}\|\in T-I\|,
$$

then $\sin T=\sqrt{1-\cos ^{2} T}$. The quantity $\mu_{1}(T)$ has numerous applications in numerical analysis as well as statistics and econometrics (see [5, 6, 8]). A vector $f$ for which the infimum in (1.1) is attained is called an antieigenvector of $T$. The first total antieigenvalue of an operator $T$ is defined to be

$$
\left|\mu_{1}\right|(T)=\inf _{T x \neq 0} \frac{|(T f, f)|}{\|T f\|\|f\|}=\inf _{\substack{T x \neq 0 \\\|f\|=1}} \frac{|(T f, f)|}{\|T f\|} .
$$

The quantity $\left|\mu_{1}\right|(T)$ is also denoted by $|\cos | T$. A vector $f$ for which the infimum in (1.3) is attained is called a total antieigenvector of $T$. For an operator $T$, we also define the quantity $\cos _{I}(T)$ by

$$
\cos _{I}(T)=\inf _{\substack{T f \neq 0 \\\|f\|=1}} \frac{\operatorname{Im}(T f, f)}{\|T f\|}
$$


and call it the imaginary cosine of $T$. The angle between a pair of operators $A$ and $B$ can be measured by looking at quantities

$$
\begin{gathered}
\cos (T, S)=\mu_{1}(T, S)=\inf _{\substack{A x \neq 0 \\
B X \neq 0}} \frac{\operatorname{Re}(T f, S f)}{\|T f\|\|S f\|}, \\
|\cos |(T, S)=\left|\mu_{1}\right|(T, S)=\inf _{\substack{A x \neq 0 \\
B X \neq 0}} \frac{|(T f, S f)|}{\|T f\|\|S f\|} .
\end{gathered}
$$

We call $\mu_{1}(T, S)$ the joint antieigenvalue for $T$ and $S$, and $\left|\mu_{1}\right|(T, S)$ is called the total joint antieigenvalue for $T$ and $S$. The quantities $\mu_{1}(T, S)$ and $\left|\mu_{1}\right|(T, S)$ are also denoted by $\cos (T, S)$ and $|\cos |(T, S)$, respectively. A vector $f$ for which the infimum in (1.5) is attained is called a joint antieigenvector for $T$ and $S$. A vector $f$ for which the infimum in (1.6) is attained is called a joint total antieigenvector for $T$ and $S$. Likewise, the quantity $\cos _{I}(T, S)$ is defined by

$$
\cos _{I}(T, S)=\inf _{\substack{A x \neq 0 \\ B X \neq 0}} \frac{\operatorname{Im}(T f, S f)}{\|T f\|\|S f\|} .
$$

The author and Gustafson have studied $\mu_{1}(T)$ and $\left|\mu_{1}\right|(T)$ for normal operators on finite- and infinite-dimensional spaces (see [9, 10, 12, 13]). Also, in [11], the author has studied $\mu_{1}(T, S)$, where $S$ and $T$ are two operators belonging to the same closed normal subalgebra of $B(H)$. Note that $\mu_{1}(T)=\mu_{1}(T, I)$ and $\left|\mu_{1}\right|(T)=\left|\mu_{1}\right|(T, I)$, where $I$ is the identity operator. Our objective in this paper is to establish some inequalities among these trigonometric functions using the Gram determinant between three vectors.

2. Operator trigonometry. Recall that any invertible operator $T$ on a Hilbert space is a product of the form $T=U P$, where $U$ is a unitary operator and $P$ is a positive operator (polar decomposition). On a finite, dimensional Hilbert space, every operator is the product of a unitary operator $U$ and a positive operator $P$. For a positive operator $P$, we know that $\mu_{1}(P)=2 \sqrt{m M} /(m+M)$, where $m$ and $M$ are the smallest and largest eigenvalues of $P$, respectively. This was first proved by Gustafson in [2] and later independently by Krein. On the other hand, for a unitary operator $U$, it is easy to see that $\mu_{1}(U)=\inf \left\{\cos \theta: e^{i \theta} \in \sigma(T)\right\}$ (see [13]). It is however impossible to express $\mu_{1}(T)$ in terms of $\mu_{1}(P)$ and $\mu_{1}(U)$. In the following, we will establish some inequalities among trigonometric functions of composite operators and their components. One may use Theorem 2.1 with $A=U$ and $B=P$ to establish an inequality between trigonometric functions of $T, U$, and $P$ if $T$ has a polar decomposition $T=U P$. Our work here is based on the fact that for any three vectors $x_{1}, x_{2}$, and $x_{3}$ in a Hilbert space, the Gram determinant

$$
G\left(x_{1}, x_{2}, x_{3}\right)=\operatorname{det}\left[\begin{array}{lll}
\left(x_{1}, x_{1}\right) & \left(x_{2}, x_{1}\right) & \left(x_{3}, x_{1}\right) \\
\left(x_{1}, x_{2}\right) & \left(x_{2}, x_{2}\right) & \left(x_{3}, x_{2}\right) \\
\left(x_{1}, x_{3}\right) & \left(x_{2}, x_{3}\right) & \left(x_{3}, x_{3}\right)
\end{array}\right]
$$

is nonnegative. 
THEOREM 2.1. If the numerical ranges of operators $A$ and $B$ are subsets of the first quadrant, then

$$
\begin{gathered}
1+2\left(\cos B \cos A B \cos (A B, B)+\cos B \cos _{I} A B \cos _{I}(A B, B)+\cos _{I} B \cos _{I} A B \cos (A B, B)\right) \\
\geq|\cos |^{2} B+|\cos |^{2} A B+|\cos |^{2}(A B, B)+2 \cos A B \cos _{I} B \cos _{I}(A B, B) .
\end{gathered}
$$

Proof. Let $x$ be any unit vector and let $G$ be the Gram determinant function defined by (2.1). Let $(B x /\|B x\|, A x /\|A x\|)=a+b i,(A B x /\|A B x\|, A x /\|A x\|)=c+d i$, and $(A B x /\|A B x\|, B x /\|B x\|)=e+f i$. Then we have $(A x /\|A x\|, A x /\|A x\|)=1,(B x /\|B x\|$, $B x /\|B x\|)=1,(A B x /\|A B x\|, A B x /\|A B x\|)=1,(A x /\|A x\|, B x /\|B x\|)=a-b i$, $(A x /\|A x\|, A B x /\|A B x\|)=a c-d i$, and $(B x /\|B x\|, A B x /\|A B x\|)=e-f i$. Therefore

$$
G\left(\frac{A x}{\|A x\|}, \frac{B x}{\|B x\|}, \frac{A B x}{\|A B x\|}\right)=\operatorname{det}\left[\begin{array}{ccc}
1 & a+b i & c+d i \\
a-b i & 1 & e+f i \\
c-d i & e-f i & 1
\end{array}\right] .
$$

Direct computations show that

$$
\operatorname{det}\left[\begin{array}{ccc}
1 & a+b i & c+d i \\
a-b i & 1 & e+f i \\
c-d i & e-f i & 1
\end{array}\right]=\begin{aligned}
& -e^{2}-f^{2}-a^{2}+2 a e c+2 a f d \\
& -b^{2}-2 c f b+2 b e d-c^{2}-d^{2} .
\end{aligned}
$$

Since the Gram determinant $G(A x /\|A x\|, B x /\|B x\|, A B x /\|A B x\|)$ is nonnegative, we have

$$
1-e^{2}-f^{2}-a^{2}+2 a e c+2 a f d-b^{2}-2 c f b+2 b e d-c^{2}-d^{2} \geq 0
$$

which implies

$$
1+2(a e c+a f d+b d e) \geq\left(a^{2}+b^{2}\right)+\left(c^{2}+d^{2}\right)+\left(e^{2}+f^{2}\right)+2 c f b .
$$

If we substitute the values of $a, b, c, d, e$, and $f$ in (2.6), we have

$$
\begin{aligned}
1+2( & \frac{\operatorname{Re}(B x, x)}{\|B x\|} \frac{\operatorname{Re}(A B x, x)}{\|A B x\|} \frac{\operatorname{Re}(A B x, B x)}{\|A B x\|\|B x\|} \\
& +\frac{\operatorname{Re}(B x, x)}{\|B x\|} \frac{\operatorname{Im}(A B x, x)}{\|A B x\|} \frac{\operatorname{Im}(A B x, B x)}{\|A B x\|\|B x\|} \\
& \left.+\frac{\operatorname{Im}(B x, x)}{\|B x\|} \frac{\operatorname{Im}(A B x, x)}{\|A B x\|} \frac{\operatorname{Re}(A B x, B x)}{\|A B x\|\|B x\|}\right) \\
\geq & \left(\frac{|(B x, x)|}{\|B x\|}\right)^{2}+\left(\frac{|(A B x, x)|}{\|A B x\|}\right)^{2}+\left(\frac{|(A B x, B x)|}{\|A B x\|\|B x\|}\right)^{2} \\
& +2 \frac{\operatorname{Re}(A B x, x)}{\|A B x\|} \frac{\operatorname{Im}(B x, x)}{\|B x\|} \frac{\operatorname{Im}(A B x, B x)}{\|A B x\|\|B x\|} .
\end{aligned}
$$

Now inequality (2.2) follows from inequality (2.7) and the properties of the infimum. 
REMARK 2.2. In [7], Gustafson and Rao used the Gram determinant to find a superset for the numerical range of the product of two bounded operators $A$ and $B$.

THEOREM 2.3. If the numerical ranges of operators $A$ and $B$ are subsets of the first quadrant, then

$$
\begin{gathered}
1+2\left(\cos A \cos B \cos (A, B)+\cos A \cos _{I} B \cos _{I}(A, B)+\cos _{I} A \cos _{I} B \cos (A, B)\right) \\
\geq|\cos |^{2} A+|\cos |^{2} B+|\cos |^{2}(A, B)+2 \cos B \cos _{I} A \cos _{I}(A, B) .
\end{gathered}
$$

Proof. Let $x$ be any unit vector. Using the nonnegativity of the Gram determinant

$$
G\left(x, \frac{A x}{\|A x\|}, \frac{B x}{\|B x\|}\right)
$$

and computations similar to those carried out in Theorem 2.1, we have

$$
\begin{aligned}
1+2( & \frac{\operatorname{Re}(x, A x)}{\|A x\|} \frac{\operatorname{Re}(x, B x)}{\|B x\|} \frac{\operatorname{Re}(A x, B x)}{\|A x\|\|B x\|} \\
& +\frac{\operatorname{Re}(x, A x)}{\|A x\|} \frac{\operatorname{Im}(x, B x)}{\|B x\|} \frac{\operatorname{Im}(A x, B x)}{\|A x\|\|B x\|} \\
& \left.+\frac{\operatorname{Im}(x, A x)}{\|A x\|} \frac{\operatorname{Im}(x, B x)}{\|B x\|} \frac{\operatorname{Re}(A x, B x)}{\|A x\|\|B x\|}\right) \\
\geq & \left(\frac{|(x, A x)|}{\|A x\|}\right)^{2}+\left(\frac{|(x, B x)|}{\|B x\|}\right)^{2}+\left(\frac{|(A x, B x)|}{\|A x\|\|B x\|}\right)^{2} \\
& +2 \frac{\operatorname{Re}(x, B x)}{\|B x\|} \frac{\operatorname{Im}(x, A x)}{\|A x\|} \frac{\operatorname{Im}(A x, B x)}{\|A x\|\|B x\|} .
\end{aligned}
$$

Now inequality (2.8) follows from inequality (2.10) and the properties of infimum.

THEOREM 2.4. Let $A, B$, and $C$ be three operators whose numerical ranges are subsets of the first quadrant, then

$$
\begin{aligned}
1+2 \cos (A, B) \cos (A, C) \cos (B, C)+2 \cos (A, B) \cos _{I}(A, C) \cos _{I}(B, C) \\
+2 \cos _{I}(A, B) \cos _{I}(A, C) \cos (B, C) \\
\geq|\cos |^{2}(A, B)+|\cos |^{2}(A, C)+|\cos |^{2}(B, C) \\
+2 \cos (A, C) \cos _{I}(A, B) \cos _{I}(B, C) .
\end{aligned}
$$

Proof. Let $x$ be any unit vector. Using the nonnegativity of the Gram determinant

$$
G\left(\frac{A x}{\|A x\|}, \frac{B x}{\|B x\|}, \frac{C x}{\|C x\|}\right)
$$


and computations similar to those carried out in Theorem 2.1, we have

$$
\begin{aligned}
1+2 & \frac{\operatorname{Re}(A x, B x)}{\|A x\|\|B x\|} \frac{\operatorname{Re}(A x, C x)}{\|A x\|\|C x\|} \frac{\operatorname{Re}(B x, C x)}{\|B x\|\|C x\|}+2 \frac{\operatorname{Re}(A x, B x)}{\|A x\|\|B x\|} \frac{\operatorname{Im}(A x, C x)}{\|A x\|\|C x\|} \frac{\operatorname{Im}(B x, C x)}{\|B x\|\|C x\|} \\
& +2 \frac{\operatorname{Im}(A x, B x)}{\|A x\|\|B x\|} \frac{\operatorname{Im}(A x, C x)}{\|A x\|\|C x\|} \frac{\operatorname{Re}(B x, C x)}{\|B x\|\|C x\|} \\
\geq & \left(\frac{|(A x, B x)|}{\|A x\|\|B x\|}\right)^{2}+\left(\frac{|(A x, C x)|}{\|A x\|\|C x\|}\right)^{2}+\left(\frac{|(B x, C x)|}{\|B x\|\|C x\|}\right)^{2} \\
& +2 \frac{\operatorname{Re}(A x, C x)}{\|A x\|\|C x\|} \frac{\operatorname{Im}(A x, B x)}{\|A x\|\|B x\|} \frac{\operatorname{Im}(B x, C x)}{\|B x\|\|C x\|} .
\end{aligned}
$$

Now inequality (2.11) follows from inequality (2.13) and the properties of infimum.

For positive operators $A$ and $B$, we have $\cos _{I} A=\cos _{I} B=0$. We also have $|\cos | A=$ $\cos A,|\cos | B=\cos B$. Therefore, inequalities (2.2) and (2.8) have simpler forms for positive operators.

COROLlARY 2.5. For positive operators $A, B$

$$
\begin{aligned}
1+2 & {\left[\cos B \cos A B \cos (A B, B)+\cos B \cos _{I} A B \cos _{I}(A B, B)\right] } \\
& \geq \cos ^{2} B+|\cos |^{2} A B+|\cos |^{2}(A B, B), \\
1+2 & \cos A \cos B \cos (A, B) \geq \cos ^{2} A+\cos ^{2} B+|\cos |^{2}(A, B) .
\end{aligned}
$$

Inequality (2.15) is particularly useful in generalizing the Kantorovich inequality. As we mentioned before, for a positive operator $T$, we have $\cos T=2 \sqrt{m M} /(m+M)$, where $m$ and $M$ are the smallest and the largest eigenvalues of $A$, respectively. This leads to the inequality

$$
\frac{(T x, x)}{\|T x\|\|x\|} \geq \frac{2 \sqrt{m M}}{m+M} \quad \forall x
$$

or

$$
\frac{(T x, x)^{2}}{\|T x\|^{2}\|x\|^{2}} \geq \frac{4 m M}{(m+M)^{2}} \quad \forall x .
$$

Inequality (2.16) (or its equivalent (2.17)) is known as the Kantorovich inequality.

THEOREM 2.6. Let $A$ be a positive operator with the smallest eigenvalue $m_{1}$ and the largest eigenvalue $M_{1}$. Let $B$ be another positive operator with the smallest eigenvalue $m_{2}$ and the largest eigenvalue $M_{2}$. Then

$$
\begin{aligned}
& \frac{4 \sqrt{\left(m_{1} M_{1} m_{2} M_{2}\right)}-\left(M_{1}-m_{1}\right)\left(M_{2}-m_{2}\right)}{\left(m_{2}+M_{2}\right)\left(m_{1}+M_{1}\right)} \\
& \leq|\cos |(A, B) \leq \frac{4 \sqrt{\left(m_{1} M_{1} m_{2} M_{2}\right)}+\left(M_{1}-m_{1}\right)\left(M_{2}-m_{2}\right)}{\left(m_{2}+M_{2}\right)\left(m_{1}+M_{1}\right)} .
\end{aligned}
$$


Proof. Since $|\cos |(A, B) \geq \cos (A, B)$ by (2.15) we have $1+2 \cos A \cos B|\cos |(A, B) \geq$ $\cos ^{2} A+\cos ^{2} B+|\cos |^{2}(A, B)$, which implies

$$
\begin{aligned}
& 1-\cos ^{2} A-\cos ^{2} B+\cos ^{2} A \cos ^{2} B \\
& \quad \geq|\cos |^{2}(A, B)-2 \cos A \cos B|\cos |(A, B)+\cos ^{2} A \cos ^{2} B .
\end{aligned}
$$

The last inequality is equivalent to

$$
[|\cos |(A, B)-\cos A \cos B]^{2} \leq 1-\cos ^{2} A-\cos ^{2} B+\cos ^{2} A \cos ^{2} B
$$

which implies

$$
\begin{aligned}
& -\sqrt{\left(1-\cos ^{2} A-\cos ^{2} B+\cos ^{2} A \cos ^{2} B\right)} \\
& \quad \leq|\cos |(A, B)-\cos A \cos B \leq \sqrt{\left(1-\cos ^{2} A-\cos ^{2} B+\cos ^{2} A \cos ^{2} B\right)} .
\end{aligned}
$$

Hence we have

$$
\begin{aligned}
& \cos A \cos B-\sqrt{\left(1-\cos ^{2} A-\cos ^{2} B+\cos ^{2} A \cos ^{2} B\right)} \\
& \quad \leq|\cos |(A, B) \leq \cos A \cos B+\sqrt{\left(1-\cos ^{2} A-\cos ^{2} B+\cos ^{2} A \cos ^{2} B\right)} .
\end{aligned}
$$

If we substitute $\cos A=2 \sqrt{m_{1} M_{1}} /\left(m_{1}+M_{1}\right)$ and $\cos B=2 \sqrt{m_{2} M_{2}} /\left(m_{2}+M_{2}\right)$ in $\sqrt{\left(1-\cos ^{2} A-\cos ^{2} B+\cos ^{2} A \cos ^{2} B\right)}$ and simplify, we obtain

$$
\sqrt{\left(1-\cos ^{2} A-\cos ^{2} B+\cos ^{2} A \cos ^{2} B\right)}=\frac{\left(M_{1}-m_{1}\right)\left(M_{2}-m_{2}\right)}{\left(m_{2}+M_{2}\right)\left(m_{1}+M_{1}\right)} .
$$

Also note that $\cos A \cos B=4 \sqrt{\left(m_{1} M_{1} m_{2} M_{2}\right)} /\left(m_{2}+M_{2}\right)\left(m_{1}+M_{1}\right)$.

EXAMPLE 2.7. Assume $A$ is a positive operator with the smallest eigenvalue $m_{1}=1$ and the largest eigenvalue $M_{1}=18$. Assume also $B$ is a positive operator with the smallest eigenvalue $m_{2}=2$ and the largest eigenvalue $M_{2}=3$. Then

$$
\begin{aligned}
& \frac{4 \sqrt{\left(m_{1} M_{1} m_{2} M_{2}\right)}-\left(M_{1}-m_{1}\right)\left(M_{2}-m_{2}\right)}{\left(m_{2}+M_{2}\right)\left(m_{1}+M_{1}\right)}=\frac{24}{95} \sqrt{3}-\frac{17}{95}=0.25862, \\
& \frac{4 \sqrt{\left(m_{1} M_{1} m_{2} M_{2}\right)}-\left(M_{1}-m_{1}\right)\left(M_{2}-m_{2}\right)}{\left(m_{2}+M_{2}\right)\left(m_{1}+M_{1}\right)}=\frac{24}{95} \sqrt{3}+\frac{17}{95}=0.6165
\end{aligned}
$$

and hence $0.25862 \leq|\cos |(A, B) \leq 0.6165$.

REMARK 2.8. Suppose $A$ is an operator with the smallest eigenvalue $m$ and the largest eigenvalue $M$, and consider $|\cos |(A, I)$ where $I$ is the identity operator. Note that for the identity operator both the smallest and the largest eigenvalues are 1 . The values 
of the expressions $\left(4 \sqrt{\left(m_{1} M_{1} m_{2} M_{2}\right)}-\left(M_{1}-m_{1}\right)\left(M_{2}-m_{2}\right)\right) /\left(m_{2}+M_{2}\right)\left(m_{1}+M_{1}\right)$ and $\left(4 \sqrt{\left(m_{1} M_{1} m_{2} M_{2}\right)}-\left(M_{1}-m_{1}\right)\left(M_{2}-m_{2}\right)\right) /\left(m_{2}+M_{2}\right)\left(m_{1}+M_{1}\right)$ are both equal to $2 \sqrt{m M} /$ $(m+M)$ for $m_{1}=m, M_{1}=M, m_{2}=1$, and $M_{2}=1$. Hence in this case $|\cos (A, I)|=$ $\cos (A, I)=2 \sqrt{m M} /(m+M)$. This is consistent with $\cos A=2 \sqrt{m M} /(m+M)$.

ACKNOWLEDGMent. Partially supported by a grant from Indiana University East.

\section{REFERENCES}

[1] K. Gustafson, The angle of an operator and positive operator products, Bull. Amer. Math. Soc. (N.S.) 74 (1968), 488-492.

[2] _ A min-max theorem, Notices Amer. Math. Soc. 15 (1968), 699.

[3] _ Positive (noncommuting) operator products and semi-groups, Math. Z. 105 (1968), 160-172.

[4] _ Antieigenvalue inequalities in operator theory, Inequalities III (Calif, 1969) (O. Shisha, ed.), Academic Press, New York, 1972, pp. 115-119.

[5] __ An extended operator trigonometry, Linear Algebra Appl. 319 (2000), no. 1-3, 117135.

[6] _ Operator trigonometry of statistics and econometrics, Linear Algebra Appl. 354 (2002), 141-158.

[7] K. Gustafson and D. Rao, Numerical range and accretivity of operator products, J. Math. Anal. Appl. 60 (1977), no. 3, 693-702.

[8] __ Numerical Range. The Field of Values of Linear Operators and Matrices, Universitext, Springer-Verlag, New York, 1997.

[9] K. Gustafson and M. Seddighin, Antieigenvalue bounds, J. Math. Anal. Appl. 143 (1989), no. 2, 327-340.

[10]_ A note on total antieigenvectors, J. Math. Anal. Appl. 178 (1993), no. 2, 603-611.

[11] M. Seddighin, On the joint antieigenvalues of operators on normal subalgebras, in preparation.

[12] _ Antieigenvalues and total antieigenvalues of normal operators, J. Math. Anal. Appl. 274 (2002), no. 1, 239-254.

[13] _ Optimally rotated vectors, Int. J. Math. Math. Sci. 2003 (2003), no. 63, 4015-4023.

Morteza Seddighin: Mathematics Department, Indiana University East, Richmond, IN 473741289, USA

E-mail address: mseddigh@indiana.edu 


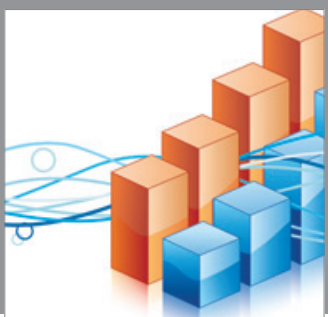

Advances in

Operations Research

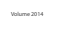

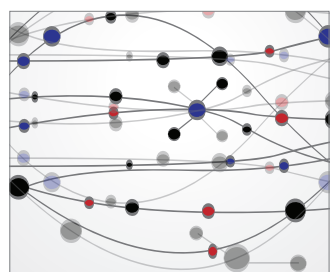

\section{The Scientific} World Journal
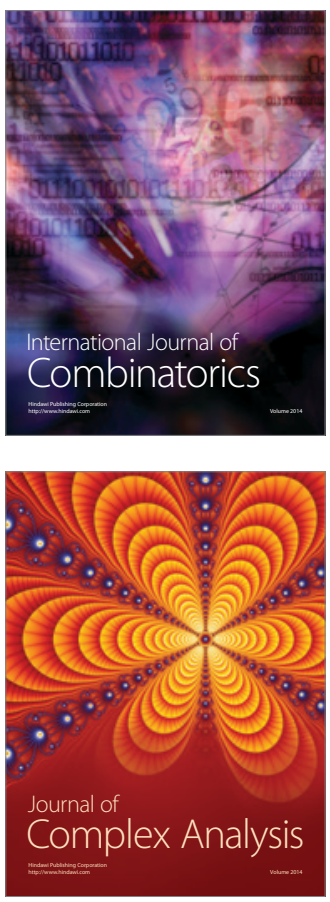

International Journal of

Mathematics and

Mathematical

Sciences
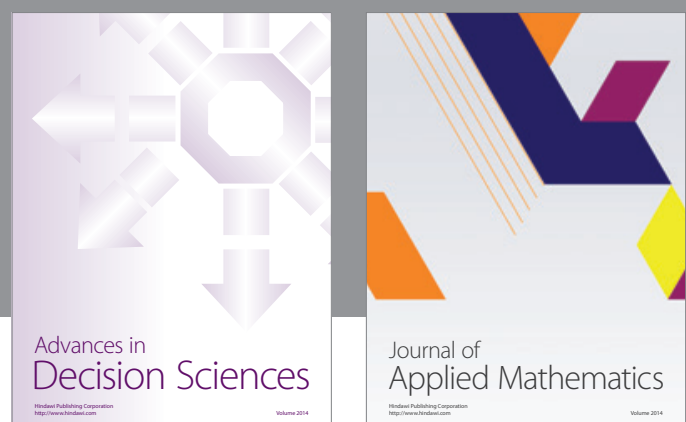

Journal of

Applied Mathematics
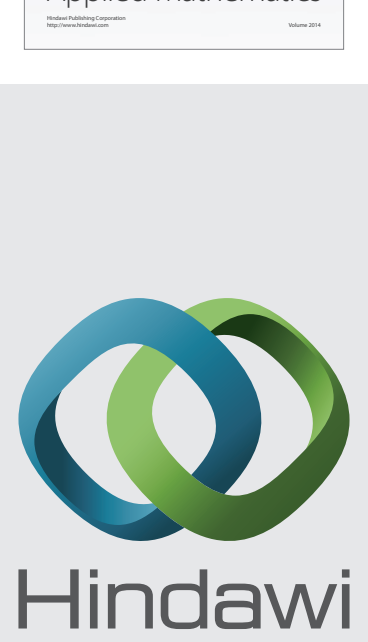

Submit your manuscripts at http://www.hindawi.com
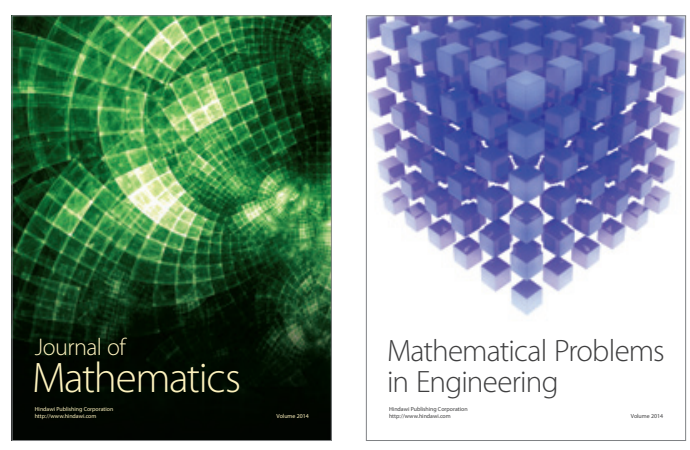

Mathematical Problems in Engineering
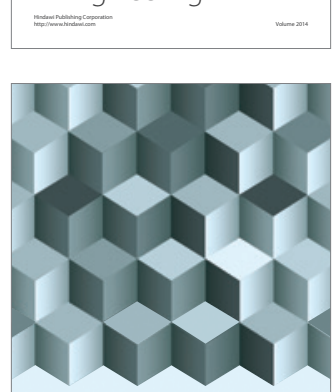

Journal of

Function Spaces
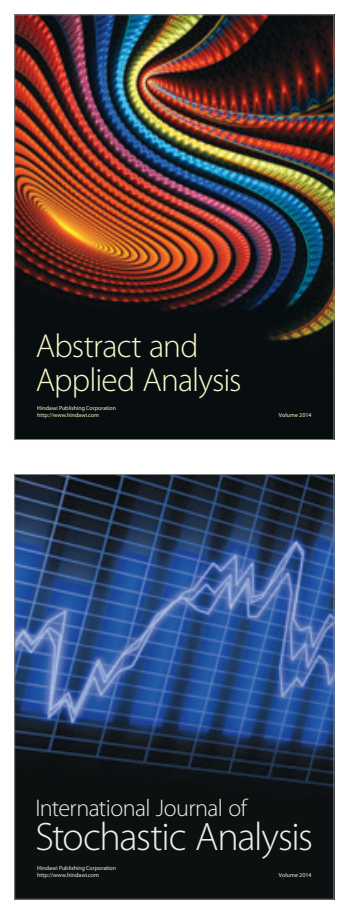

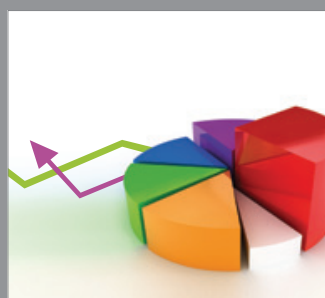

ournal of

Probability and Statistics

Promensencen
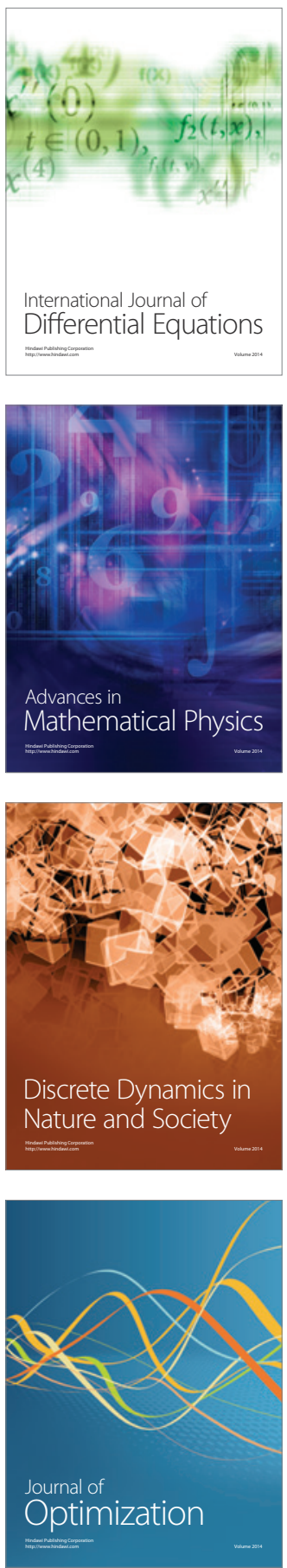